Pathologe 2008 · [Suppl 2] 29:123-128

DOI 10.1007/s00292-008-1045-8

(C) Springer Medizin Verlag 2008

M. Wagner ${ }^{1} \cdot$ T. Breiner ${ }^{2} \cdot$ T. Betz $^{3}$. I. Bernhardt ${ }^{3} \cdot$ N. Pütz ${ }^{4}$. F. Weichert ${ }^{5}$.

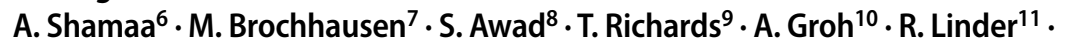
C.A. Landes ${ }^{12}$

${ }^{1}$ Institut für Allgemeine und Spezielle Pathologie, Universität des Saarlandes, Campus Homburg Saar

${ }^{2}$ Institut für Informatik, SRH Hochschule Heidelberg

${ }^{3}$ AG Biophysik, Universität des Saarlandes, Campus Saarbrücken

${ }^{4}$ Anatomisches Institut, Universität des Saarlandes, Campus Homburg Saar

${ }^{5}$ Informatik VII, Technische Universität Dortmund

${ }^{6}$ Department of Oral Biology, Minia University, Minia, Ägypten

${ }^{7}$ Institute for Formal Ontology and Medical Information Science (IFOMIS), Universität des Saarlandes, Campus Saarbrücken

${ }^{8}$ Department of Oral Surgery, Mansoura University, Mansoura, Ägypten

${ }^{9}$ University of the West Indies, Kingston, Jamaika

${ }^{10}$ Institut für Angewandte Mathematik, Universität

des Saarlandes, Campus Saarbrücken

${ }^{11}$ Institut für Medizinische Informatik, Universität zu Lübeck

${ }^{12}$ Klinik für Mund-, Kiefer- und Plastische Gesichtschirurgie,

Universitätsklinikum Frankfurt am Main

\title{
Virtuelles Gewebe
}

\section{Quaoaring}

Da es sich bei der vorliegenden Arbeit um einen interdisziplinären Ansatz handelt, ist es unabdingbar, scheinbar triviale Begriffe zu erläutern, um auch fachfremden Leserinnen und Lesern die notwendigen Zusammenhänge näher zu bringen. Der hier beschriebene Ansatz geht zudem mit einer eigenständigen, relativ umfangreichen Terminologie einher, die einerseits zwar inhaltliche Bezüge zur Nomina Anatomica Veterinaria bzw. zur Terminologia Anatomica aufweist, andererseits aber auch einige, oftmals nichtpolyseme Homonyme besitzt, wie z. B. den Begriff Biofeld. Eine entsprechende Ontologie ist noch zu erarbeiten.

Ungeübte Betrachter mögen es schwierig finden, Ergebnisse mathematischer Modelle lediglich anhand von Zahlen oder Graphen korrekt zu interpretieren. An reale Mikroskopie angelehnte Visualisierungskonzepte liefern derzeit grob vereinfachte, stark schematisierte Abbildungen, mit deren Hilfe sich Fragen zu zyto- oder histomorphologisch relevanten Details auch von histopathologisch ausge- bildeten Auswertern nicht immer zufriedenstellend beantworten lassen. Um Histologen und Histopathologen u. a. den Zugang zu Ergebnissen geeigneter Simulationen erleichtern zu können, sollten künstliche Zellen und Gewebe daher bedarfsgerecht, d. h. soweit wie möglich fotorealistisch, visualisiert werden. Histologische und histopathologische Expertise könnte dann, in Analogie zu realen Laborexperimenten, eine wesentliche Instanz der Qualitätskontrolle entsprechender systembiologischer Simulationen bilden.

Histologisches Expertenwissen ist derzeit eines der Hauptinstrumente medizinbiologischer Qualitätskontrolle. Im Gegensatz hierzu sind zyto- bzw. histomorphologische Bezüge bei der Verifikation systembiologischer Simulationsergebnisse bislang noch dramatisch unterrepräsentiert. Daher wird hier exemplarisch ein Ansatz zur Herstellung virtueller Zell- und Gewebetypen beschrieben, mit dessen Hilfe sich eines Tages Ergebnisse systembiologischer Experimente auch zytologischer oder histologischer
Expertise zuführen lassen sollten. Unsere Arbeitsgruppe plant, erfolgversprechende Verfahren zukünftig auch beispielsweise in Mikrochirurgiesimulationen aus dem Bereich der Mund-, Kiefer- und Gesichtschirurgie zu integrieren.

Erste Schritte auf dem Weg zu virtuellem Gewebe beginnen mit der Herstellung virtueller Zellen. Als zelluläre Komponente eignen sich Erythrozyten adulter Vertreter der Spezies Homo sapiens („National Center for Biotechnology Information Taxonomy Identifier“ " 8] 9606) aufgrund ihres Aufbaus in besonderer Weise für die Umsetzung des hier vorgestellten Ansatzes. Sie können in nullter Näherung als Membransäcke aufgefasst werden, die mit einer Hämoglobinlösung gefüllt sind und weder einen Zellkern noch Mitochondrien, Ribosomen oder ein endoplasmatisches Retikulum enthalten. Diese Reduktion legt nahe, dass das Erscheinungsbild der Zellmembran innerhalb des Untersuchungszeitraumes zumindest nicht durch Transskription oder Translation von Membranproteinen in nennens- 


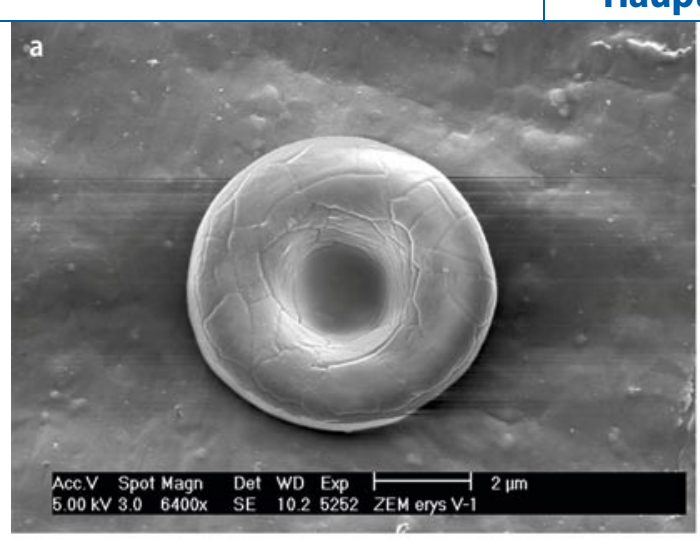

\section{Hauptreferate: Systempathologie des Gewebes}
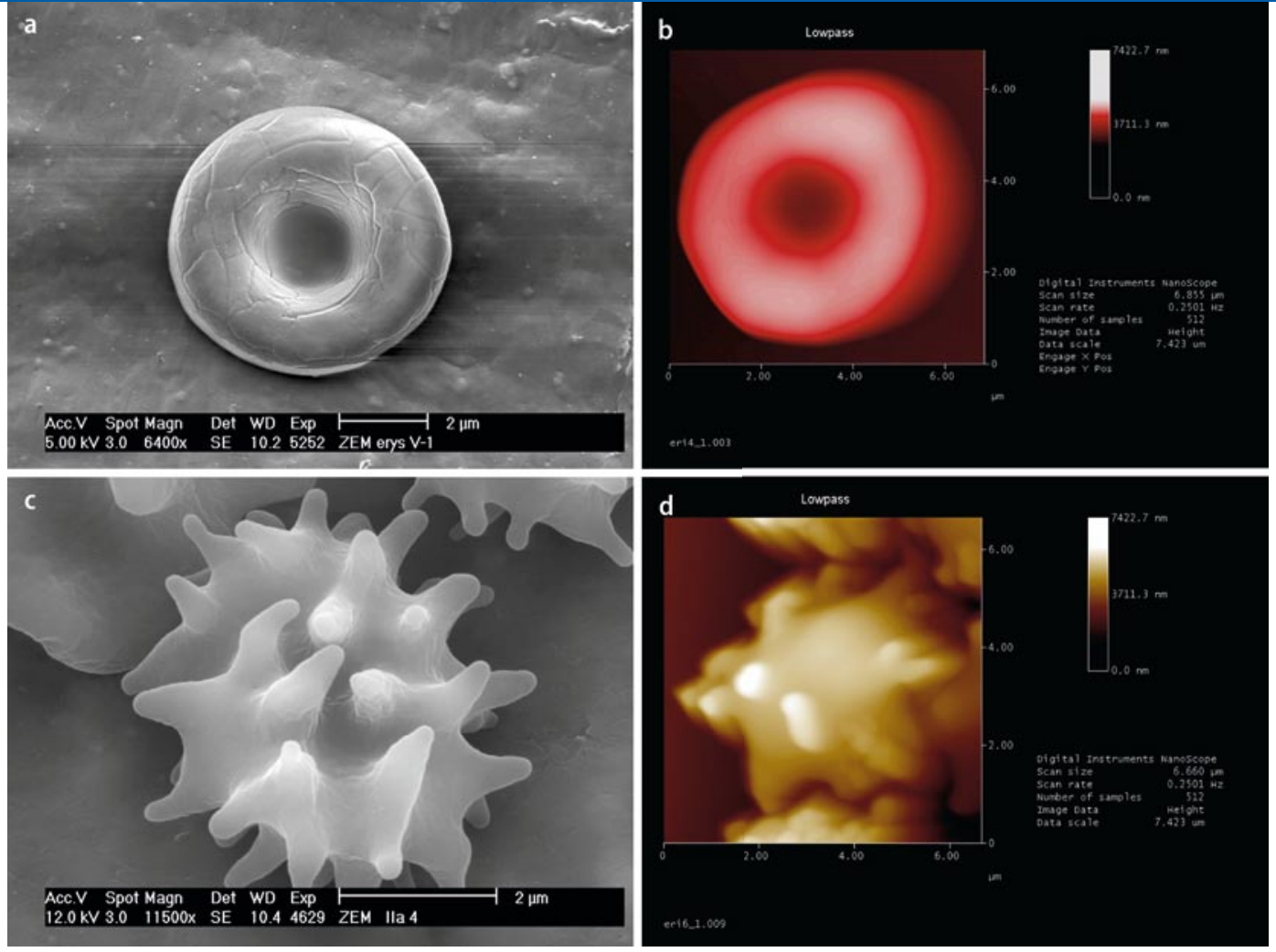

Abb. $1 \Delta$ Experimentell induzierte Formvarianten realer Erythrozyten. Zahlreiche Vertiefungen überziehen die Oberfläche dieses diskozytär geformten realen Erythrozyten. Während diese Darstellungen mit den Ergebnissen der Quaoaring-Technologie korrespondierten, war die Modellierung der unten abgebildeten echinozytären Geometrie nur unzureichend möglich. Echinozytär geformte reale Erythrozyten besitzen ausgeprägte Spiculae. Die Protrusionen der Zellmembran waren mit diesem Ansatz nur bedingt realitätsnah nachzubilden. a Diskozytenform; Rasterelektronenmikroskopie (Vergr. 6400:1).

b Erythrozyt; Atomic-Force-Mikroskopie (Scan-Range: 6,885×6,885 $\mu \mathrm{m}$ ). c Echinozytenform; Rasterelektronenmikroskopie (Vergr. 11.500:1).d Echinozytenform; Atomic-Force-Mikroskopie (Scan-Range: 6,660×6,660 $\mu \mathrm{m}$ )

werter Größenordnung nachhaltig modifiziert wurde. Rote Blutkörperchen stellen daher ein geeignetes Modell für das zunächst erarbeitete Konzept der Visualisierung von Zellmembranen dar.

In der so genannten Diskozytenform sind Erythrozyten bikonkav, haben einen Durchmesser von rund $7,5 \mu \mathrm{m}$, eine Dicke von etwa $2 \mu \mathrm{m}$, eine Membranoberfläche von ungefähr $140 \mu^{2}$ und ein Volumen von etwa $95 \mu \mathrm{m}^{3}$. Formveränderungen können wie bei Sphärozyten mit oder wie bei Stomatozyten- oder Echinozytenformen ohne nennenswerte Veränderung des Zellvolumens auftreten $[2,3]$. Da Erythrozyten als Membransäcke mit einer Hämoglobinfüllung dem Konzept der so genannten hydropneumatischen Einheit (synonym: Pneu) nahestehen, das metaphorisch durch einen „wassergefüllten Ballon" repräsentiert wird, wurde ein neues Konzept aus dem Bereich der Computergraphik gewählt, welches dieser Vereinfachung entspricht: das Quaoaring.
Des Begriff Quaoar ([5, 7]; [kwa:wa:r] S[kwa:owa:] oder [q'wa:wa:]) stammt aus der Sprache kalifornischer Ureinwohner, der Tongva, und beschreibt eine allumfassende Schöpfungskraft. Mit Hilfe der Quaoaring-Technologie können deformierbare Objekte mit vergleichsweise geringem Aufwand ausgehend von einer hydropneumatischen Einheit modelliert und animiert werden. Obwohl die Quaoaring-Technologie bislang zur dreidimensionalen Darstellung mehrzelliger tierischer Organismen verwendet wird, ist die Nutzung der Grundidee als Visualisierungsbasis von einzelnen Zellen, speziell von Erythrozyten, neu.

Als ideale hydropneumatische Einheit wird im Zusammenhang mit der Quaoaring-Technologie ein Pneu bezeichnet, auf den keinerlei Nettokräfte einwirken, sodass das Ergebnis kugelförmig ist. Um andere Formen zu erzeugen, muss mit Hilfe mathematischer Ansätze der Einfluss deformierender Kräfte auf diesen compu- tergenerierten Körper simuliert werden. Dessen Außengrenzen werden durch eine netzartige Oberflächenstruktur (synonym: „Mesh“) definiert, die aus Dreiecken mit den zugehörigen Eckpunkten besteht, den so genannten „Vertices“. So bietet sich Quaoaring als Modellierungsbasis von Zellen an, da es physikalische Interaktionen zwischen Intra- und Extrazellularraum, wie Osmose, sowie Eigenschaften von Membranen, beispielsweise einer Phospholipidmembran, oder stabilisierenden und verspannenden Strukturen, z. B. dem Zytoskelett, unterstützt. Darüber hinaus ist die Quaoaring-Technologie für die Darstellung beispielsweise von Metazoa, Bilateria und Coelomata geeignet. Hüllen und Füllungen hydropneumatischer Einheiten können selbstverständlich mehr oder weniger viskös und in ein computergeneriertes Medium eingebettet sein, mit dem sie beispielsweise mechanisch interagieren können. Hierbei erweist sich zumindest theoretisch als 
Vorteil, dass es neben einer intuitiv-interaktiven Modellierung der Hüllform auch eine prozedurale Animation von Deformationsprozessen unterstützt. Kernstück ist in diesem Zusammenhang die unmittelbare visuelle Rückkopplung auf die Eingabe von Wachstumskorrekturbefehlen, auf deren Basis per Augenschein sowohl quantitative als auch qualitative Modifikationen interaktiv vorgenommen werden können. Die Modellierung stellt im Konzept des Quaoaring somit keinen von der Visualisierung abgekoppelten Formerzeugungsprozess dar. Vielmehr stehen hier Simulation und Visualisierung in enger Verbindung zueinander.

Für die Deformation hydropneumatischer Einheiten ist hilfreich, dass sie eine fiktive interne Achse besitzen. Diese als Zentrallinie bezeichnete imaginäre Hilfslinie verbindet die gegenüberliegenden Pole der hydropneumatischen Einheit miteinander, die durch Intersektion der Achse mit der Hülle als Start- und Endpunkt der Zentrallinie definiert sind. Diese Zentrallinie wird durch die Interpolation zwischen verschiedenen Knotenpunkten (synonym: „Nodes“) erzeugt, die im Falle des computergenerierten Erythrozyten äquidistant wie Perlen an eine Perlenkette aneinandergereiht sind. Mit Hilfe dieser Nodes kann jeder Eckpunkt $P$ des Polygons im Mesh über dessen Abstand zur Zentrallinie und die Position des Node auf dieser Linie bestimmt werden. Dies gilt allerdings nur, solange ein bestimmter Winkel bekannt ist. Dieser öffnet sich zwischen derjenigen Geraden, die durch den Node und den Eckpunkt $P$ geht, und jener Schnittebene des computergenerierten Erythrozyten, die sich zwischen der X- und der Y-Achse im globalen kartesischen Koordinatensystem aufspannt. Durch das Konstrukt der Zentrallinie kann jeder Eckpunkt der Polygone im Mesh dadurch beschrieben werden, dass ein Koordinatensystem, das im Kontext der Quaoaring-Technologie als „Biologisches Koordinatensystem “ bezeichnet wird, entlang der Zentrallinie ausgerichtet ist.

Die Position eines beliebigen Eckpunktes $P$ eines Polygons im Mesh kann im Biologischen Koordinatensystem jeweils durch einen so genannten Biovektor $\tau$ mit dem Format $\tau:=\left(f_{\mathrm{R}} ; f_{\mathrm{A}} ; f_{\mathrm{H}}\right)$ beschrieben werden. Bezogen auf den Abstand zwischen Start- und Endpunkt der Zentrallinie, repräsentiert $f_{\mathrm{R}}$ hierbei den relativen Abstand entlang dieser Linie, ausgehend von deren Startpunkt. Dabei wird derjenige Punkt, der im betreffenden Abstand auf der Zentrallinie liegt, als Hilfspunkt $P^{c}$ bezeichnet. Am Programmanfang wird ein beliebiger Normalenvektor, der eine Senkrechte, ausgehend von der Zentrallinie, definiert, zur Orientierung des Koordinatensystems festgelegt. Die Komponente $f_{\mathrm{A}}$ beschreibt dann den im Bogenmaß anzugebenden Winkel zwischen diesem Normalenvektor und der Linie, die zwischen $P^{\text {‘ }}$ und $P$ verläuft. Der Term $f_{\mathrm{H}}$ schließlich steht für den relativen Abstand zwischen der Zentrallinie und dem Mesh, ausgehend von $P^{r}$. Dementsprechend hat der Eckpunkt $P$ eines Polygons des Mesh per definitionem stets den Wert 1.

Die Parameter für das so genannte Biofeld $S$ einer hydropneumatischen Einheit kann im Biologischen Koordinatensystem durch den Term $S:=\left(c_{\mathrm{R}} ; c_{\mathrm{A}} ; c_{\mathrm{H}} ; r_{\mathrm{R}}\right.$; $\left.r_{\mathrm{A}} ; r_{\mathrm{H}}\right)$ beschrieben werden, wobei die ersten 3 Parameter den Mittelpunkt und die 3 letzen Parameter die dreidimensionale Ausdehnung jeweils im Biologischen Koordinatensystem repräsentieren. Hierbei steht $r_{\mathrm{R}}$ für den fraktionalen longitudialen Radius relativ zur Gesamtlänge der hydropneumatischen Einheit, $r_{\mathrm{A}}$ für die halbe orthogonale Ausdehnung (gemessen in rad) und $r_{\mathrm{H}}$ für die radiale Länge relativ zum Abstand zwischen der Zentrallinie und der Hülle, der entlang derjenigen Normalen zur Zentrallinie bestimmt wird, die den Mittelpunkt von $S$ durchstößt.

Wird die Simulation einer Deformation als eine lokale Beeinflussung der Eckpunkte der Polygone in einem Mesh aufgefasst, kann die Position von $P$ durch den oben beschriebenen Ansatz kontrolliert verändert werden. So kann die computergenerierte flüssigkeitsgefüllte Kugel mit Hilfe mathematisch modellierter äußerer Krafteinwirkung beispielsweise entlang der Zentrallinie verkürzt werden. Dies führt unter dem Primat der Volumenkonstanz in Verbindung mit der elastischen Außenhülle des computergenerierten Hohlkörpers zwangsläufig zu dessen Verformung. Diese resultiert aus einer Ausbreitung der hydropneumatischen Einheit in all jene Richtungen, die von der
Pathologe 2008 · [Suppl 2] 29:123-128

DOI 10.1007/s00292-008-1045-8

(C) Springer Medizin Verlag 2008

M. Wagner · T. Breiner · T. Betz $\cdot$ I. Bernhardt · N. Pütz · F. Weichert · A. Shamaa . M. Brochhausen - S. Awad - T. Richards . A. Groh · R. Linder · C.A. Landes Virtuelles Gewebe. Quaoaring

Zusammenfassung

Es gibt mannigfaltige Wege, virtuelles Gewebe herzustellen. Erste Schritte dorthin können über die Herstellung virtueller Zellen führen. Eine "Quaoaring" genannte Methode wurde genutzt, um artifizielle Erythrozyten sowohl der Diskozyten- als auch der Echinozytenform zu erzeugen. Die Ergebnisse wurden mit Daten aus der Rasterelektronenmikroskopie und der „Atomic-Force-Mikroskopie" verglichen. Quaoaring konnte hierbei insbesondere bei Objekten, die eine Echinozytenform haben sollten, nicht überzeugen.

Schlüsselwörter

Systembiologie - Erythrozyt · Atomic-ForceMikroskopie · Elektronenmikroskopie · Visualisierung

\section{Virtual tissue. Quaoaring}

\section{Abstract}

Virtual tissue can be generated by employing various methods. First steps en route to virtual tissue may encompass the generation of virtual cells. One such approach termed Quaoaring was applied to produce artificial erythrocytes and these were both discocyte and echinocyte in shape. The results were subsequently compared with data gleaned from scanning electron microscopy and atomic force microscopy. Quaoaring has, however, proved to be unsuccessful in creating convincing objects, particularly those which should be echinocytic in appearance.

Keywords

Systems biology - Erythrocyte - Atomic force microscopy · Electron microscopy · Visualisation 


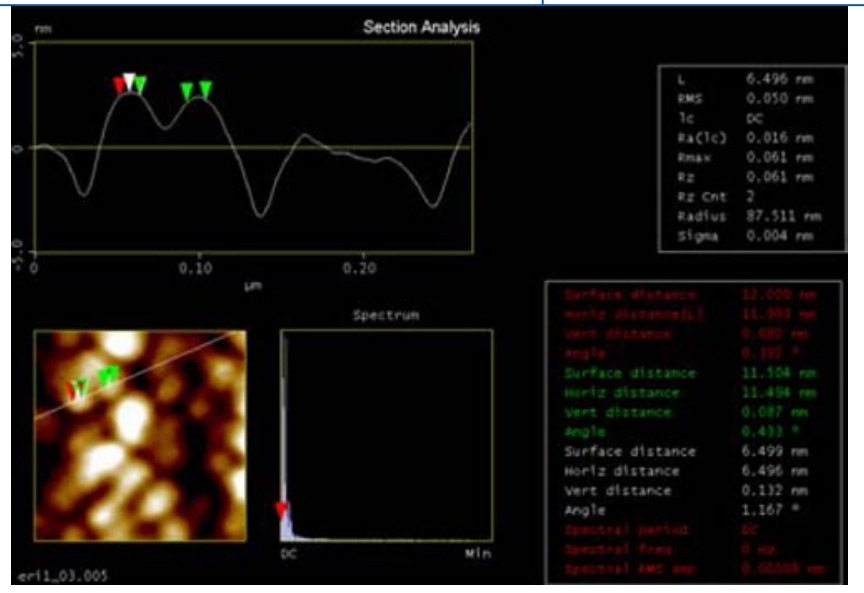

\section{Hauptreferate: Systempathologie des Gewebes}

Abb. $2 \triangle$ Zelloberfläche der Diskozytenform. Mit Hilfe der Atomic-ForceMikroskopie wurden entlang der Z-Achse Interferenzen zwischen verschiedenen Komponenten des Oberflächenprofils der Erythrozyten detektiert. Die Oberfläche der computergenerierten Zellen wurde daher mit entsprechend konfigurierten virtuell nanostrukturellen Repräsentanten der quantitativen Analyseergebnisse bestückt. Atomic-Force-Mikroskopie;

Scan-Range: etwa 0,25×0,25 $\mu \mathrm{m}$

Krafteinwirkung nicht direkt erfasst wurden.

Um die Oberfläche einer hydropneumatischen Einheit im Zusammenhang mit der Quaoaring-Technologie mit Feinstrukturen versehen zu können, wurde eine Technik eingeführt, die als „Hydrostatisches Bump-Mapping" bezeichnet wird und eine Spezialisierung und Weiterentwicklung so genannter morphischer Felder darstellt. Der Term morphisches Feld beschreibt im Kontext der QuaoaringTechnologie ein zunächst nicht präzise definiertes Areal, das entweder in oder um ein hierarchisch geschachteltes so genanntes morphisches Objekt herum gelegen ist. Während bei einem einfachen morphischen Feld das Einwirken einer einzigen großen Kraft auf die pneumatische Einheit modelliert wird, werden beim Hydrostatischen Bump-Mapping viele kleinere Kräfte auf ein Punktfeld $T_{\mathrm{i}, \mathrm{j}}$ der Oberfläche appliziert. Dabei wird der Betrag der jeweiligen Kräfte $F_{\mathrm{i}, \mathrm{j}}$ modifiziert. Durch diese Krafteinwirkungen werden die jeweiligen Deformationsfaktoren $f_{\mathrm{S}}$ an den Oberflächenpunkten $T_{\mathrm{i}, \mathrm{j}}$ indirekt über die Wechselwirkung von $F_{\mathrm{i}, \mathrm{j}}$ mit dem inneren hydrostatischen Druck $P$ verändert.

Der algorithmische Ablauf des Quaoaring ist dadurch gekennzeichnet, dass in wesentlichen Anteilen des Ansatzes die Anweisungsblöcke so lange ausgeführt werden, bis die Kriterien für ein Programmende erfüllt sind. In einem ers- ten Schritt wird eine ideale hydropneumatische Einheit, also ein undeformierter Ausgangspneu mit einem definierten Ausgangsvolumen, mit Hilfe eines Polygonnetzes erzeugt. Danach werden verschiedene Kräfte in Form von morphischen Feldern auf die Hülle der hydropneumatischen Einheit angewendet, die diese im Rahmen einer Simulation verformen. Neben äußerlich einwirkenden Kräften können Größenveränderungen natürlich auch durch Anheben oder Absenken des Volumens des Mediums im Innenraum des Hohlkörpers erzeugt werden. Das Erscheinungsbild der hydropneumatischen Einheit wird somit durch computergenerierte Kraftanwendungen und andere Maßgaben interaktiv in die gewünschten Bahnen gelenkt.

Um eine hydropneumatische Einheit durch eine spezifische computergenerierte Kraft zu deformieren, muss zuerst jener Abschnitt der Hülle definiert werden, auf den ein verformender Effekt ausgeübt werden soll, die so genannte Biofläche. Anschließend wird der Kraftbetrag angegeben. Alternativ kann direkt auch die maximale Auslenkung des Deformationswulstes $\omega$ spezifiziert werden, die aus der Kraftanwendung resultieren würde. Die Auslenkung wird dabei relativ zum aktuellen Abstand zwischen Zentrallinie und Hülle angegeben, wobei entlang jenem Normalenvektor der Zentrallinie gemessen wird, der den Mittelpunkt der Biofläche durchstößt. Die Größe $\omega$ kann mit Hilfe eines Quotienten berechnet werden, der aus der Kraft $F$, dem internen hydrostatischen Druck $P$ und jener Oberfläche $A$ besteht, auf die $F$ appliziert wird (Formel 1).

$\omega=\frac{F}{P \cdot A}-1$

Der Wert für die Oberfläche $A$ kann aus der Spezifikation der Biofläche extrahiert werden, während $P$ und $F$ entweder als experimentell ermittelte Parameter oder als berechnete Werte aus einer Simulation in die Formel eingehen können. Werden das so genannte Biofeld $S$ und der Konvexitätsparameter $d$ in den Rechenprozess eingeführt, kann ein morphisches Feld durch $F:=(S ; \omega ; d)$ beschrieben werden. Mit Hilfe so genannter zweidimensionaler „togglebarer Blob-Funktionen“ lassen sich u. a. deformierende Gebilde auf der Oberfläche der computergenerierten Erythrozyten erzeugen [1]. Der Begriff „toggle“ steht dafür, dass sich der Kurvenverlauf der Funktion über den Parameter $d$ modifizieren lässt. Über die Wahl des Wertes für $d$ kann die Ausprägung der Konvexität des Deformationswulstes modifiziert werden, wobei ein niedriger Wert die Deformation stumpf und dickwulstig werden lässt, während ein großer Wert eine spitze, eher stachelförmige Konvexität erzeugt.

Im Kontext der Quaoaring-Technologie werden Deformationen mit Hilfe der zweidimensionalen togglebaren BlobFunktionen angenähert, wobei für derartige Veränderungen hydrostatische Eigenschaften berücksichtigt werden müssen, die den Gesetzen der Physik nachempfunden sind. Will man eine hydropneumatische Einheit dahingehend modifizieren, dass sie die Form eines computergenerierten Erythrozyten annimmt, muss das Einwirken von Kräften implementiert werden, die eine hydrostatisch plausible Verformung des computergenerierten Hohlkörpers bei gleichbleibendem Volumen induzieren. Um den Eingangswert für die Blob-Funktion $B(r, d)$ an einem bestimmten Oberflächenpunkt $\tau:=\left(f_{\mathrm{R}} ; f_{\mathrm{A}} ; f_{\mathrm{H}}\right)$ $\mathrm{zu}$ ermitteln, muss eine $\Gamma$-Distanzfunktion erstellt werden (Formel 2).

$\Gamma(\tau, S)=\sqrt{\left(\frac{f_{R}-c_{R}}{r_{R}}\right)^{2}+\left(\frac{f_{A}-c_{A}}{r_{A}}\right)^{2}+\left(\frac{f_{H}-c_{H}}{r_{H}}\right)^{2}}$ 
Hierdurch wird die skalare Entfernung zwischen dem Punkt $\tau$ innerhalb des Biofeldes $S:=\left(c_{\mathrm{R}} ; c_{\mathrm{A}} ; c_{\mathrm{H}}=1 ; r_{\mathrm{R}} ; r_{\mathrm{A}} ; r_{\mathrm{H}}=0\right)$ zum Zentrum von $S$ errechnet, wobei das Ergebnis für das entsprechende morphische Feld $F$ mit den relativen Auslenkungen $\omega$ in Beziehung gesetzt wird. Für ein gegebenes morphisches Feld, das durch $F:=(S ; \omega ; d)$ repräsentiert ist, kann somit der Deformationsfaktor $\Theta(\tau, F)$ des Punktes $\tau:=\left(f_{\mathrm{R}} ; f_{\mathrm{A}} ; f_{\mathrm{H}}\right)$ durch die Formel 3:

$$
\begin{aligned}
\Theta(\tau, F) & =\left\{\begin{array}{cc}
\frac{(K-1)^{2}}{(1+d \cdot K)} \cdot \omega & K<1 \\
0 & K \geqslant 1
\end{array},\right. \\
K & :=\left(\frac{f_{R}-c_{R}}{r_{R}}\right)^{2}+\left(\frac{f_{A}-c_{A}}{r_{A}}\right)^{2}+\left(\frac{f_{H}-c_{H}}{r_{H}}\right)^{2}
\end{aligned}
$$

berechnet und der resultierende Deformationsfaktor $f_{\mathrm{S}}$ am Oberflächenpunkt $\tau$ durch die Formel 4:

$$
f_{S}=\sum_{i=1}^{n} \Theta\left(\tau, F_{i}\right)
$$

für diejenige Menge von $n$ morphischen Feldern $F_{\mathrm{i}}(i=1, \ldots, n)$ bestimmt werden, die auf $\tau$ einen topologischen Einfluss haben.

Im Rahmen der Quaoaring-Technologie wird u. a. automatisch eine Datensammlung von so genannten „Hydrostatischen Bump-Mapping-Texturen“ angelegt. Es besteht zudem eine Reihe von Normen und Richtlinien, die das Quaoaring u. a. hinsichtlich Datentyp, Kompressionsart, Auflösung, Farbgebung auch jenseits von Projektgrenzen kompatibel macht. Bei der Programmierung können Zellmembranoberflächen und deren sich teilweise überlagernde Vorwölbungen und Vertiefungen jeweils als separate morphische Felder angesprochen werden. Durch dieses Konstrukt findet die potenzielle Überlappung der Daten aus der „Atomic-Force-Mikroskopie“ ein Korrelat in der potenziellen Überlappung der zugehörigen morphischen Felder.

\section{Material und Methoden}

Die virtuelle Repräsentation der roten Blutkörperchen wurde durch Modellierung einer idealen hydropneumatischen Einheit initiiert, die mit computergenerierten Kräften unter Einhaltung der Vo- lumenkonstanz bis zum Entstehen der gewünschten Morphologie verformt wurde. Als Referenz dienten neben morphometrischen Daten realer Erythrozyten auch rasterelektronenmikroskopische Aufnahmen sowie Daten aus der Atomic-ForceMikroskopie. Exemplarisch wurden mittels Atomic-Force-Mikroskopie 30 willkürlich gesetzte Areale im Nanometerbereich analysiert, da mutmaßlich etwa 28 Messungen in dieser Größenordnung ausreichen, um von einem Atomic-Force-Mikroskop einen Datensatz zu erhalten, der es erlaubt, die gesamte Oberfläche eines Erythrozyten zu rekonstruieren [9]. Die vorgelagerte laborexperimentelle Aufarbeitung der Erythrozyten ist bereits an anderer Stelle detailliert dargestellt worden [4].

Zur Herstellung der computergenerierten Erythrozytenhülle wurde eine hydropneumatische Einheit in Form eines geschlossenen Polygonnetzes mit einer Auflösung von $1200 \times 18$ oo Polygonen vorbereitet. Um die bikonkave Diskozytenform zu erzeugen, wurden mit Hilfe des Design-Patterns „Squeeze“ auf 2 Antipoden der zunächst kugelförmigen hydropneumatischen Einheit jeweils zentripedale Kraftfelder simuliert, die die Antipoden zusammenzogen. Das Biologische Koordinatensystem zur Darstellung der Diskozytenform wurde so orientiert, dass die Zentrallinie identisch mit der Symmetrielinie des Erythrozyten war, die sich zwischen den beiden tiefsten Einbuchtungen aufspannte. Die Diskozytenform wurde durch eine hydropneumatische Einheit (Auflösung radial: 1200 Vertices; longitudinal: 900 Vertices) mit 2 großflächigen morphischen Feldern (Design-Pattern: "Squeeze") gebildet, die durch $F_{1}=\left(S_{1} ; \omega_{1}\right.$; $\left.d_{1}\right)$ und $F_{2}=\left(S_{2} ; \omega_{2} ; d_{2}\right)$ repräsentiert wurden. Die Biofeldparameter des ersten morphischen Feldes lagen bei $S_{1}:=\left(c_{\mathrm{R}}=0 ; c_{\mathrm{A}}=0\right.$; $\left.c_{\mathrm{H}}=1 ; r_{\mathrm{R}}=0,572 ; r_{\mathrm{A}}=4 \pi ; r_{\mathrm{H}}=0\right)$, die des zweiten bei $S_{2}:=\left(c_{\mathrm{R}}=1 ; c_{\mathrm{A}}=0 ; c_{\mathrm{H}}=1 ; r_{\mathrm{R}}=0,572\right.$; $\left.r_{\mathrm{A}}=4 \pi ; r_{\mathrm{H}}=0\right)$. Als Auslenkungsparameter wurde jeweils $\omega_{1}=\omega_{2}=-0,723$ gewählt, während die Konvexitätsparameter $d_{1}$ und $d_{2}$ jeweils auf 1,21 gesetzt wurden. Unter anderem wurde die so genannte Technik der Fraktalen Netze angewendet, um jene mit einem Gauß-Unschärfefilter (Unschärfedistanz $=4,5$ Pixel) behandelte Hydrostatische Bump-Mapping-Textur zu

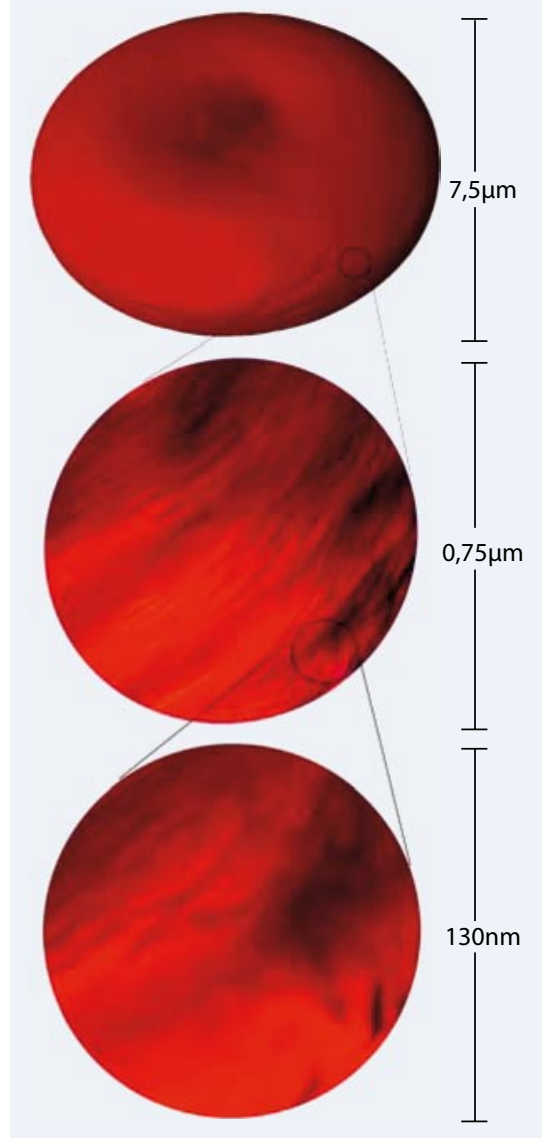

Abb. $3 \Delta$ Computergenerierte Diskozytenform eines Erythrozyten. Bereits in der kleinsten Vergrößerungsstufe zeigt sich zusätzlich zum reinen Hydrostatischen Bump-Mapping die identisch skalierte so genannte Image-Textur auf der Hüllform des computergenerierten Erythrozyten. Diese Interferenz repräsentiert Daten aus der Atomic-Force-Mikroskopie, zu der sich Details in den willkürlich gewählten virtuellen Ausschnittsvergrößerungen zeigen. Quaoaring-Technologie

applizieren, die mit einer Auflösung von 4048×4048 Pixeln die Messdaten der Atomic-Force-Mikroskopie repräsentierte.

\section{Ergebnisse}

Sowohl anhand der Messergebnisse der Atomic-Force-Mikroskopie als auch in den rasterelektronenmikroskopischen Aufnahmen besaßen die realen Erythrozyten der Kontrollpräparate entsprechend den vorgeschalteten Versuchsbedingungen entweder eine Diskozyten- oder eine Echinozytenform (- Abb. 1 a-d, - Abb. 2, 3). Obschon es sich hierbei also lediglich um 2 Ausprägungen eines einzigen Objekts handelt, gelang es nicht, die beiden Geomet- 


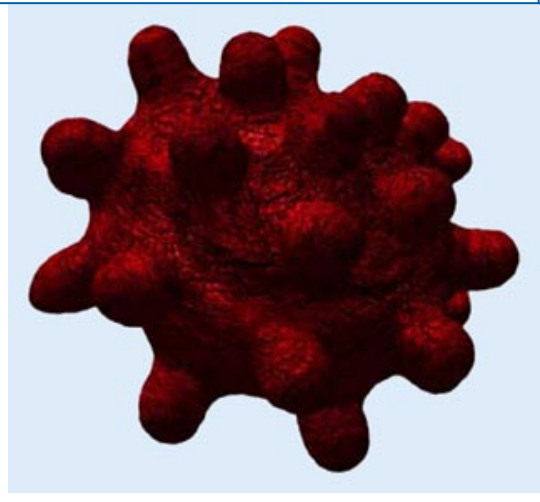

Abb. $4 \Delta$ Versuch einer computergenerierten Darstellung der Echinozytenform eines Erythrozyten. Die computergenerierte Echinozytenform ist ohne Ablation individueller Helligkeitsund Farbschattierungen der Image-Texturen abgebildet. Diese wurden zusätzlich zum reinen Hydrostatischen Bump-Mapping auf die Hüllform des Erythrozyten appliziert, um durch verschiedene Schattierungsnuancen Unterschiede im Höhenprofil optisch besser hervorheben zu können. So wurden geometrieassoziierte Artefakte der Oberflächenstrukturen deutlich, weshalb im Vergleich mit den Vorlagen aus der Rasterelektronenmikroskopie und der AtomicForce-Mikroskopie nicht nur die Repräsentation der Spiculae unbefriedigend erscheint.

Quaoaring-Technologie

rien durch multifokale Verformung einer singulären hydropneumatischen Einheit mit Hilfe der Quaoaring-Technologie in zufriedenstellender Weise ineinander zu überführen. Stattdessen bedurfte es zur zumindest groben Approximierung der Geometrie einer Echinozytenform mit Spiculae eines Stadiums mit $\Delta A_{\mathrm{o}}=0$,018 [6] der Apposition untergeordneter hydropneumatischer Einheiten (synonym: Subpneu, „Bulges“) an eine übergeordnete hydropneumatische Einheit (synonym: Hauptpneu, „Mainpneu“, Rumpfpneu). Trotz elaborierter Simulation unterschiedlicher Druckverhältnisse entsprach die mit einer derartigen Modellierung morphischer Felder assoziierte Modifikation der Geometrie eines Pneus nicht den Erwartungen an eine möglichst plausible Darstellung ( $\bullet$ Abb. 4). Die Generierung der künstlichen Echinozytenform hatte zudem ausgeprägte geometrische Verziehungen und Verwerfungen der applizierten Hydrostatischen BumpMapping- und Image-Texturen zur Folge. In dem hier nachgewiesenen Ausmaß traten diese Artefakte bei der Texturierung der Diskozytenform nicht auf.

\section{Diskussion}

Exemplarisch wurde ein Visualisierungskonzept mit potenziell großer Mächtigkeit vorgestellt, welches gleichermaßen virtuelle Prokaryonten und Eukaryonten (und darunter nicht nur Zellen tierischen Ursprungs) darzustellen vermag. Es sollte untersucht werden, ob das so genannte Quaoaring eine realitätsnahe, möglichst hochauflösende Darstellung der Verformung von künstlichen Zellen unterstützt. Im Rahmen dieser Studie wurde die Quaoaring-Technologie weltweit zum ersten Mal als Visualisierungsbasis für Erythrozyten eingesetzt und mit Ergebnissen der Atomic-Force-Mikroskopie verknüpft. Prinzipiell unterstützt die hier vorgestellte Methodenkombination daher die Visualisierung von Zellmembranen mit bis zu molekularer oder gar atomarer Auflösung. Eine derart detaillierte Darstellung von Oberflächencharakteristika kann dazu beitragen, beispielsweise Simulationsergebnisse zur Rezeptordichte zu veranschaulichen. Allerdings sind zahlreiche weitergehende Untersuchungen nötig, um den lediglich morphologisch charakterisierten Membranstrukturen die zugehörigen biochemischen Korrelate zuordnen zu können. Bis dies geschehen ist, bleibt die hier vorgestellte Darstellungsform als „proof of principle“ symbolhaft, was bei kritischer Analyse den zugrundeliegenden Aufwand fragwürdig erscheinen lässt. Eine zufriedenstellende Modellierung und Darstellung der Zellverformung gelang nicht. Somit vermag die Quaoaring-Technologie in ihrer aktuellen Form nicht, Histologen und Histopathologen den Zugang zu Ergebnissen entsprechender systembiologischer mathematischer Modelle zu erleichtern. Daher scheint diese Methode auch für einen Einsatz im Bereich der virtuellen Mikrochirurgie nicht geeignet zu sein, wie sie derzeit von unserer Arbeitsgruppe für mund-, kiefer- und gesichtschirurgische Operationssimulationen vorbereitet wird.

Die Quaoaring-Technologie kam im Gegensatz zu weiteren Ansätzen unserer Arbeitsgruppe ohne vorgelagerte Segmentierung aus (s. die Beiträge von Weichert et al. und Groh et al. in dieser Ausgabe von „Der Pathologe“).
Fazit für die Praxis

Die Quaoaring-Technologie ist vergleichsweise aufwendig und führte nicht zu den von der Arbeitsgruppe angestrebten Ergebnissen. Ihre Anwendung gehört daher (wie derzeit noch viele Ansätze aus der Systembiologie) in den Bereich der Grundlagenforschung. Die Quaoaring-Technologie vermag es in ihrer aktuellen Form möglicherweise nicht, Histologen und Histopathologen den Zugang zu Ergebnissen komplexer systembiologischer mathematischer Modelle in geeigneter Weise zu erleichtern. Auch spielt sie zu Recht derzeit in der täglichen pathologisch-anatomischen Diagnostik keine Rolle.

\section{Korrespondenzadresse}

\section{PD Dr. M. Wagner}

Insitut für Allgemeine und Spezielle Pathologie, Universitätsklinikum des Saarlandes, Campus Homburg Saar, Gebäude 25, 66421 Homburg-Saar

Interessenkonflikt. Erklärung: Die Autoren versichern, dass keine finanziellen oder persönlichen Beziehungen zu Dritten gegeben sind, deren Interessen vom Beitragsinhalt positiv oder negativ betroffen sein könnten. Es besteht keine derartige Beeinflussung.

\section{Literatur}

1. Baranoski GVG, Rokne J (2002) An efficient and controllable blob function. J Graph Tools 4: 41-44

2. Bessis M (1972) Red cell shapes. An illustrated classification and its rationale. Nouv Rev Fr Hematol 12: 721-745

3. Bessis M, Weed RI (1973) The structure of normal and pathologic erythrocytes. Adv Biol Med Phys 14: 35-91

4. Betz T, Bakowsky U, Müller MR et al. (2007) Conformational change of membrane proteins leads to shape changes of red blood cells. Bioelectrochemistry 70: 122-126

5. Jewitt DC, Luu J (2004) Crystalline water ice on the Kuiper belt object (50000) Quaoar. Nature 432: 731-733

6. Mukhopadhyay R, Lim HWG, Wortis M (2002) Echinocyte shapes: bending, stretching and shear determine spicule shape and spacing. Biophys J 82: 1756-1772

7. Stevenson DJ (2004) Planetary science: volcanoes on Quaoar? Nature 432: 681-682

8. Wheeler DL, Chappey C, Lash AE et al. (2000) Database resources of the national center for biotechnology information. Nucleic Acids Res 28: 10-14

9. Zhang PC, Bai C, Huang YM et al. (1995) Atomic force microscopy study of fine structures of the entire surface of red blood cells. Scanning Microsc 9: 981-989 (Discussion 1009-1010) 